\title{
Adding glimepiride to current insulin therapy increases high-molecular weight adiponectin levels to improve glycemic control in poorly controlled type 2 diabetes
}

Chun-Jun $\mathrm{Li}^{1,2}$, Jing-Yun Zhang ${ }^{1,2}$, De-Min $\mathrm{Yu}^{1,2^{*}}$ and Qiu-Mei Zhang ${ }^{1,2^{*}}$

\begin{abstract}
Background: To observe the efficacy and safety of adding glimepiride to established insulin therapy in poorly controlled type 2 diabetes (T2D) and to assess the relationship of changes in the serum high-molecular weight (HMW) adiponectin levels and glycemic control after glimepiride treatment.

Methods: Fifty-six subjects with poorly controlled insulin-treated T2D were randomly assigned to either the glimepiride-added group (the group $A, n=29$ ) or the insulin-increasing group (the group $B, n=27$ ) while continuing current insulin-based therapy. Glycosylated hemoglobin ( $\mathrm{HbA1c}$ ) value, daily insulin dose, body weight, waist circumference, plasma lipid concentration, serum HMW adiponectin level and the number of hypoglycemic events were evaluated before and after treatment.

Results: At the end of study, insulin doses were significantly reduced, and the mean $\mathrm{HbA1c}$, fasting blood glucose (FBG) and 2-hour postprandial blood glucose (P2BG) were improved greater in the group A compared with the group B. The serum HMW adiponectin levels were significantly increased in the group A compared with the group B. Most importantly, we found that changes in $\mathrm{HbA1c}$ were inversely correlated with changes in serum HMW adiponectin in the group $A(r=-0.452, p=0.02)$.

Conclusions: Adding glimepiride to current insulin treatment led to better improvement in glycemic control with a significant smaller daily insulin dose, and the increases in the serum HMW adiponectin levels may directly contribute to improvement glycemic control.
\end{abstract}

Keywords: Glimepiride, Insulin therapy, HMW adiponectin, Type 2 diabetes

\section{Introduction}

Tight glycemic control with either intensive insulin therapy or sulfonylurea has been associated with weight gain in patients with type 2 diabetes (T2D) [1,2]. Achieving glycemic control is a critical metabolic goal because hyperglycemia contributes to the progression of T2D by adversely affecting both $\beta$-cell function and insulin sensitivity [3]. In clinical practice, achieving sustained glycemic

\footnotetext{
*Correspondence: Ii_chunjun@126.com; zhzhzhtj@126.com

'2011 Collaborative Innovation Center of Tianjin for Medical Epigenetics, Key Laboratory of Hormone and Development (Ministry of Health), Metabolic Disease Hospital, Tianjin 300070, China

${ }^{2}$ Tianjin Institute of Endocrinology, Tianjin Medical University, Tianjin 300070,
} China

lowering over time is an important aspect of therapy that is not achieved in many patients treated with large dosage of exogenous insulin combination with non-sulfonylurea drugs [4]. In addition, new treatment paradigm, aimed at reducing insulin resistance may represent a more effective treatment to decrease insulin dose. Unfortunately, most of the insulin sensitizers such as thiazolidinediones (TZDs) are associated with chronic heart failure [5]. Increasing the insulin doses or changing the insulin regimen to multiple injections of insulin could improve glycemic control. However, both endogenous hyperinsulinaemia and exogenous insulin could increase the risk of atherosclerosis and cancer [6], meanwhile some physicians have concerns 
about hypoglycemia and weight gain with intensive insulin treatment [7].

Glimepiride, a third-generation sulfonylurea (SU), exerts its effects mainly by stimulating insulin secretion but has also been shown to have extrapancreatic effect such as improvement insulin resistance [8]. Low level of adipnectin was associated with insulin resistance, obesity, and T2D [9]. Adiponectin is specifically and abundantly expressed in adipose tissue and directly sensitizes the body to insulin, which exists as three forms: a trimer of low molecular weight, a hexamer of medium molecular weight and a larger multimeric high molecular weight (HMW) form [10]. Glimepiride is reported to increase adiponectin gene expression in adipocytes [11]. And several studies have also demonstrated that glimepiride may increase in insulin sensitivity associated with increased serum adiponectinemia [12-14]. The HMW adiponectin has been the active form of the hormone and has relevant role in enhancing insulin sensitivity and in protecting against diabetes [15]. Recently, several studies have observed pioglitazone therapy significantly improving glycemic control and markedly increasing serum HMW adiponectin levels in T2D $[16,17]$. However, it is still unclear whether glimepiride treatment in T2D patients could increase serum HMW adiponectin level to improve glycemic control. And limited studies have reported that combination therapy with glimepiride and insulin could improve glycemic control and reduce insulin requirements $[18,19]$, but the mechanisms are not clear. Therefore, the present study was conducted to analyze the relationship between the degree of lowering HbAlc and serum HMW adiponectin levels and provide predictors of which patients would benefit from addition glimepiride in poorly controlled T2D subjects with insulin therapy.

\section{Materials and methods}

This study was undertaken in the out-patient setting of the Metabolic Disease Hospital of Tianjin Medical University. Subjects eligible for the study met the following criteria: T2D was defined by Chinese Diabetes Association and $\mathrm{HbA1c}$ exceeding $8 \%$ treated by large dosage of insulin (daily insulin dose more than 40 units) for at least 6 months. Subjects were excluded if they had hepatic injury (serum alanine or aspartate aminotransferase 2.5 of more times the upper-normal range), or congestive heart failure (NYHA Class III or IV) or renal damage (serum creatinine above $2.0 \mathrm{mg} / \mathrm{dl}$ ), and those already receiving sulfonylureas or insulin sensitizers such as TZDs within 6 months prior to the recruitment. Eligible subjects were explained the goals and risk of the study and gave their written informed consent before beginning the study. The study protocol was approved by the Tianjin Medical University Ethics Committee Review Board and was conducted in accordance with the Declaration of Helsinki and Good Clinical Practice guidelines. Fifty-six subjects with T2D were randomly assigned into either the glimepiride-added group (the group $A, n=29$ ) or the insulin-increasing group (the group $B, n=27$ ) while continuing based therapy.

HbA1c value, daily insulin dose, body weight, and the number of hypoglycemic events were recorded at weeks 0 , 12 and 24. Plasma lipid concentrations, serum $C$ peptide and HMW adiponectin concentration were measured at weeks 0 and 24. The glycemic control target was defined as fasting blood glucose $(\mathrm{FBG}) \leq 7.0 \mathrm{mmol} / \mathrm{L}$ and 2-hour postprandial blood glucose $(\mathrm{P} 2 \mathrm{BG}) \leq 10 \mathrm{mmol} / \mathrm{L}$. In the group A, glimepiride (Amaryl, Sanofi aventis) was initiated at the minimum dosage $1 \mathrm{mg}$ once daily and then titrated up to $4 \mathrm{mg}$ daily until the glycemic control target. In the group $\mathrm{B}$, insulin doses were increased to reach the glycemic control target. Hypoglycemic episodes and adverse events (AEs) were recorded throughout the study. Hypoglycemia was determined by the number of blood glucose readings that were below $3.9 \mathrm{mmol} / \mathrm{L}$, or occurrences of definite hypoglycemic symptoms. Hypoglycemia was considered severe when the event required third party assistance. Adverse events were classified as serious if they resulted in death, life-threatening experiences, hospitalization, or persistant of significant disability or incapacity.

In addition, we have divided the group A subjects into two sub-groups, according to the degree of HbAlc lowering (Responder, greater than $0.5 \% \mathrm{HbA} 1 \mathrm{c}$ lowering, Non-responder, less than $0.5 \% \mathrm{HbA} 1 \mathrm{c}$ lowering). Plasma total cholesterol, high-density lipoprotein (HDL) cholesterol, and triglyceride concentrations were assessed using standard enzymatic methods. HbA1c was assayed using high-performance liquid chromatography. The HMW adiponectin was measured with commercial ELISA Kit (R\&D Systems, USA).

\section{Statistical analysis}

Normally distributed data are expressed as mean \pm standard deviation and non-normally distributed data median or as numbers and percentages. Non-normally distributed data were logtransformed for use with parametric statistics. Unpaired t test was used to compare the differences in clinical characteristics between groups at baseline and after treatment assessed for significance using for the discrete or continuous data and the chi-square test for frequency distributions. The changes in HbA1c, FBG and 2 h-BG over time (at baseline and at the other 2 visits) were studied using the repeated measurements ANOVA with treatment as grouping factor. Paired $t$ tests were used to compare within-group changes. And unpaired t tests were also used to compare baseline variables between Responders and Non-responders in subjects treated with glimepiride-added. Linear regressions were performed to determine relationships between changes in serum HMW 
adiponectin levels and changes in HbA1c. The statistical analyses were performed using SPSS windows version 18.0 , and $\mathrm{p}$ value $<0.05$ was considered to be statistical significance.

\section{Results}

A total of 56 subjects with poorly controlled insulintreated T2D were recruited and completed the trial. At baseline, the two groups did not differ regarding anthropometric data, duration of diabetes and insulin treatment, BMI, waist circumference, body weight, HbA1c value, FBG, P2BG, plasma lipid concentration, daily insulin dose, frequency of insulin injections, combined antidiabetic agents, C-peptide concentration and HMW adiponectin levels. Overall, the subjects had a long duration of diabetes and poor glycemic control with large dosage of insulin. $75 \%$ of the subjects had abdominal obesity defined by Chinese Diabetes Association (Male waist circumference $\geq 90 \mathrm{~cm}$, Female waist circumference $\geq 85 \mathrm{~cm}$ ). A similar portion of subjects in the two groups were suffering from diabetic complications such as nephropathy, retinopathy and cardiovascular disease (Table 1).

During the study, mean values of HbA1c, FBG and P2BG were significantly reduced in both groups (Table 2). Repeated measurements of ANOVA on decreases in HbA1c, FBG and P2BG over the time were significant different between groups and greater in the group $A$ $(\mathrm{p}<0.01)$. The primary efficacy endpoint, the reductions in $\mathrm{HbA} 1 \mathrm{c}$ of $2.0 \%$ from baseline in the group A was significant greater than reductions of $1.6 \%$ in the group B $(\mathrm{p}<0.01)$. The target HbA1c level $7.0 \%$ achieved by 15 subjects (52\%) in the group A was larger than 8 subjects (30\%) in the group $B(p<0.05)$. The required insulin doses were reduced by $53 \%$ (from $68.6 \pm 14.6$ to $32.3 \pm 14.9$ unit/d) in the group A, while it was increased by $29 \%$ (from $66.8 \pm 13.9$ to $86.2 \pm 18.9$ unit/d) in the group B, the difference is significant. Body weight was increased significantly during the course of the study in the group B (from $67.2 \pm 15.9 \mathrm{~kg}$ to $69.8 \pm 16.5 \mathrm{~kg}, \mathrm{p}<0.05)$; however, there was no significant change in body weight in the group $\mathrm{A}$ (from $66.2 \pm 16.3$ to $66.9 \pm 16.6 \mathrm{~kg}$, p > 0.05).

There were no significant changes in plasma triglyceride, total cholesterol, LDL-cholesterol, HDL-cholesterol and plasma $\mathrm{C}$ peptide in both groups. Serum HMW adiponectin levels were markedly increased from (3.12 \pm 1.56) $\mu \mathrm{g} / \mathrm{ml}$ to $(5.86 \pm 1.62) \mu \mathrm{g} / \mathrm{ml}$ in the group $\mathrm{A}$, while no significant changes in the group B (Table 3). Changes in HbA1c were inversely associated with changes in serum HMW adiponectin in the group A $(r=-0.452$, $\mathrm{p}=0.02$ ). Reinforcing this, when we stratified patients into subgroups according to the degree of HbA1c lowering $\geq 0.5 \%$ or $0.5 \%$ : the responder group $(n=23)$ and the non-responder group $(n=6)$, there were significant greater increases in the HMW adiponectin concentrations
Table 1 Baseline characteristics of subjects in the 2 groups

\begin{tabular}{|c|c|c|}
\hline Clinical characteristics & $\begin{array}{l}\text { The group A } \\
(\mathrm{n}=29)\end{array}$ & $\begin{array}{l}\text { The group B } \\
(\mathrm{n}=27)\end{array}$ \\
\hline Age (years) & $56.8 \pm 12.3$ & $56.3 \pm 12.4$ \\
\hline Sex (Male/Female) & $15 / 14$ & $13 / 14$ \\
\hline Body weight (kg) & $66.2 \pm 16.3$ & $67.2 \pm 15.9$ \\
\hline $\mathrm{BMI}\left(\mathrm{kg} / \mathrm{m}^{2}\right)$ & $25.2 \pm 3.6$ & $25.4 \pm 3.7$ \\
\hline Waist Circumference (cm) & $93.55 \pm 10.5$ & $93.46 \pm 10.4$ \\
\hline duration of diabetes (years) & $15.6 \pm 5.7$ & $15.4 \pm 6.2$ \\
\hline Hypertention (+/-) & $22 / 7$ & $21 / 6$ \\
\hline Retinopathy(NDR/SDR/PDR) & $2 / 17 / 10$ & $2 / 16 / 9$ \\
\hline $\begin{array}{l}\text { Nephropathy(Normo-/micro-/ } \\
\text { macroalbuminuria) }\end{array}$ & $6 / 14 / 9$ & $5 / 14 / 8$ \\
\hline $\mathrm{CVD}(-/+)$ & $5 / 24$ & $5 / 22$ \\
\hline a-glucosidase inhibitors $(-/+)$ & $2 / 27$ & $1 / 26$ \\
\hline Metformin $(-/+)$ & $13 / 16$ & $14 / 13$ \\
\hline Duration of insulin treatment (years) & $9.6 \pm 3.4$ & $9.4 \pm 3.5$ \\
\hline Total insulin dose (unit/day) & $68.6 \pm 14.6$ & $66.8 \pm 13.9$ \\
\hline $\begin{array}{l}\text { Frequency of insulin injection }(2-/ 3-/-4 \\
\text { times } / d)\end{array}$ & $17 / 8 / 4$ & $16 / 7 / 4$ \\
\hline $\mathrm{HbA1c}(\%)$ & $9.3 \pm 1.5$ & $9.4 \pm 1.4$ \\
\hline $\mathrm{FBG}(\mathrm{mmol} / \mathrm{L})$ & $11.4 \pm 2.4$ & $11.3 \pm 2.6$ \\
\hline P2BG (mmol/L) & $16.8 \pm 4.4$ & $17.0 \pm 4.5$ \\
\hline Triglyceride $(\mathrm{mmol} / \mathrm{L})$ & $3.26 \pm 1.14$ & $3.28 \pm 1.16$ \\
\hline Total Cholesterol (mmol/L) & $6.23 \pm 1.41$ & $6.19 \pm 1.51$ \\
\hline HDL-Cholesterol (mmol/L) & $1.04 \pm 0.23$ & $1.12 \pm 0.21$ \\
\hline LDL-Cholesterol (mmol/L) & $4.13 \pm 1.01$ & $4.20 \pm 1.02$ \\
\hline Serum C peptide (ng/ml) & $1.06 \pm 0.68$ & $1.06 \pm 0.70$ \\
\hline HMW adiponectin $(\mu \mathrm{g} / \mathrm{ml})$ & $3.12 \pm 1.56$ & $3.22 \pm 1.54$ \\
\hline
\end{tabular}

Normally distributed data expressed as mean \pm standard deviation and non-normally distributed data expressed as median or as numbers and percentages. Non-normally distributed data were log-transformed for use with parametric statistics. BMI: body mass index; NDR: no diabetic retinopathy; SDR: simple diabetic retinopathy; PDR: proliferative diabetic retinopathy; CVD: cardiovascular disease; HbA1c: Glycosylated hemoglobin; FBG: fasting blood glucose; P2BG: 2-hour postprandial blood glucose; LDL: low-density lipoprotein; HMW adiponectin: high-molecular weight adiponectin.

in the responder group compared with the non-responder group. In addition, there were higher levels of body weight, waist circumference, HbA1c, FBG, total insulin doses and lower HMW adiponectin concentrations in the responder group compared with the non-responder group at baseline (Table 4). However, a weak but significant linear correlation was found between the baseline HbA1c values and changes in HbA1c after treatment $(r=-0.38$, $\mathrm{p}=0.04$ ), and no significant correlation was found between baseline serum HMW adiponectin and changes in HbA1c $(r=0.29, \mathrm{p}=0.15)$.

During the 24-week observation period, hypoglycemic episodes were significantly lower in the group A than in the group B ( $<<0.05)$; Moreover, significantly fewer subjects in 
Table 2 Assessment of treatments for efficacy in glycemic control in the 2 groups

\begin{tabular}{llll}
\hline Variables & $\mathbf{0}$ week & $\mathbf{1 2}$ weeks & $\mathbf{2 4}$ weeks \\
\hline HbA1c (\%) & & & \\
The group A & $9.3 \pm 1.5$ & $8.2 \pm 1.2^{*}$ & $7.3 \pm 1.3^{* *}$ \\
The group B & $9.4 \pm 1.4$ & $8.6 \pm 1.3^{*}$ & $7.9 \pm 1.3^{* \#}$ \\
FBG (mmol/L) & & & \\
The group A & $11.4 \pm 2.4$ & $7.6 \pm 1.8^{* *}$ & $6.9 \pm 1.3^{* *}$ \\
The group B & $11.3 \pm 2.6$ & $8.2 \pm 2.1^{* *}$ & $7.7 \pm 1.6^{* * \#}$ \\
P2BG (mmol/L) & & & \\
The group A & $16.8 \pm 4.4$ & $11.2 \pm 2.3^{* *}$ & $8.6 \pm 2.1^{* *}$ \\
The group B & $17.0 \pm 4.5$ & $12.4 \pm 2.3^{* *}$ & $10.8 \pm 2.2^{* * \#}$ \\
Insulin dose (unit/day) & & & \\
The group A & $68.6 \pm 14.6$ & $44.8 \pm 15.9^{* *}$ & $32.3 \pm 14.9^{* *}$ \\
The group B & $66.8 \pm 13.9$ & $79.3 \pm 14.8^{*}$ & $86.2 \pm 18.9^{* * \# \#}$ \\
Body Weight (kg) & & & \\
The group A & $66.2 \pm 16.3$ & $66.4 \pm 16.5$ & $66.9 \pm 16.6$ \\
The group B & $67.2 \pm 15.9$ & $68.3 \pm 16.0$ & $69.8 \pm 16.5^{* * \#}$ \\
\hline
\end{tabular}

Data expressed mean \pm SD; HbA1c: Glycosylated hemoglobin; FBG: fasting blood glucose; P2BG: 2-hour postprandial blood glucose; ${ }^{*} p<0.05$ vs. baseline, ${ }^{* *} p<0.01$ vs. baseline. ${ }^{\#} p<0.05$ group $A$ vs. group $B,{ }^{\# \#} p<0.01$ group $A$ vs. group $B$.

the group A experienced at least one hypoglycemic episode. Despite the occurrence of hypoglycemic episodes in the two groups, no episode was classified as severe, requiring assistance. Moreover, no AE was recorded during the study.

\section{Discussion}

In the present study, subjects with poorly controlled T2D achieved significant mean reductions in the HbA1c,

Table 3 Changes in outcome parameters after 24 weeks treatment

\begin{tabular}{|c|c|c|c|c|}
\hline \multirow[t]{2}{*}{ Variable } & \multicolumn{2}{|c|}{ The group $A(n=29)$} & \multicolumn{2}{|c|}{ The group $B(n=27)$} \\
\hline & Baseline & 24 weeks & Baseline & 24 weeks \\
\hline $\begin{array}{l}\text { Triglyceride } \\
\text { (mmol/L) }\end{array}$ & $3.26 \pm 1.14$ & 3.16 & $3.28 \pm 1.16$ & 1.15 \\
\hline $\begin{array}{l}\text { Total Cholesterol } \\
(\mathrm{mmol} / \mathrm{L})\end{array}$ & 6. & 6.18 & $6.19 \pm 1.51$ & $6.17 \pm 1.68$ \\
\hline $\begin{array}{l}\text { HDL-Cholesterol } \\
(\mathrm{mmol} / \mathrm{L})\end{array}$ & $4 \pm 0.23$ & 1.14 & 0.21 & $1.13 \pm 0.24$ \\
\hline $\begin{array}{l}\text { LDL-Cholesterol } \\
(\mathrm{mmol} / \mathrm{L})\end{array}$ & $4.13 \pm 1.01$ & $3.67 \pm 1.12$ & $4.20 \pm 1.02$ & $3.81 \pm 1.20$ \\
\hline $\begin{array}{l}\text { Serum C peptide } \\
(\mathrm{ng} / \mathrm{ml})\end{array}$ & $1.06 \pm 0.68$ & $1.18 \pm 0.66$ & $1.06 \pm 0.70$ & $1.10 \pm 0.69$ \\
\hline $\begin{array}{l}\text { HMW adiponectin } \\
\text { (ug/ml) }\end{array}$ & $3.12 \pm 1.56$ & $5.86 \pm 1.62^{*}$ & $3.22 \pm 1.54$ & $3.24 \pm 1.53^{\# \#}$ \\
\hline
\end{tabular}

Data expressed mean \pm SD; LDL: low-density lipoprotein; HMW: high-molecular weight; ${ }^{*} p<0.05$ difference compared with baseline, ${ }^{\# \#} p<0.01$ compared absolute changes between the group A and group B following 24 weeks glimepiride treatment.
FBG and P2BG with glimepiride-added treatment compared with increasing insulin doses. A significant higher percentage of subjects $(52 \%)$ in the group A had an HbAlc goal of $\leq 7 \%$ at the end of study compared with $(30 \%)$ in the group B, reflecting the greater mean reduction in the HbA1c observed in the group A. Furthermore, adding glimepiride also remarkably decreased insulin doses and offered clear advantages in terms of a reduction in the incidence of hypoglycemia and no weight gain. The most interesting findings reported here were the significant correlations between $\mathrm{HbAlc}$ lowering and increases in serum HMW adiponectin levels following 24-week glimepiride treatment $(\mathrm{r}=-0.452, \mathrm{p}=$ 0.02). We also found that serum HMW adiponectin levels increased particularly more in patients who got more than $0.5 \%$ reductions in HbA1c compared with patients who got less than $0.5 \%$, suggesting the possible role of increases in serum HMW adiponectin levels on the glimepiride-induced glycemic control.

In practice, a significant number of subjects with T2D cannot achieve tight glycemic control despite treatment with large dosage insulin combination with nonsulfonylurea drugs over time. Most of physicians are reluctant to continue to increase insulin doses or to carry out intensive insulin treatment due to the side effects associated with insulin-induced hypoglycemia and weight gain. Combinations of insulin with secondgeneration sulfonylurea derivatives, such as glibenclamide and gliclazide, have been found to offer significant improvement in glycemic control with a significantly smaller daily insulin dose [20]. SU use with insulin works because of the higher levels of insulin in the portal circulation with SU, compared to subcutaneous insulin, lowers hepatic glucose output and therefore fasting insulin better. Although many SU agents have been administered successfully with insulin, only glimepiride has been approved by the United States Food and Drug Adiministration for combination therapy. Moreover, only glimepiride has been shown not to block the beneficial effect of myocardial ischemic preconditioning that glyburide and glipizide have [21]. Furthermore, a large cohort study recently provided the clinical evidence of a trend toward an increased overall mortality risk with glyburide or glipizide versus glimepiride in those with documented cardiovascular disease [22]. Therefore, we preferred to add glimepiride to the current insulin therapy in the present study. Several clinical studies have supported that addition of glimepiride in the subjects with poorly controlled insulin-treated type 2 diabetes could improve glycemic control and reduce insulin requirements $[18,19]$. However, the underlined mechanisms are still not clear.

Insulin resistance has been the main reason for gradual worsening of glycemic control in obese T2D subjects. Obese T2D has become a big problem in Asian subjects, 
Table 4 Changes in the responders and non- responder following 24 weeks treatment

\begin{tabular}{|c|c|c|c|c|}
\hline \multirow[t]{2}{*}{ Variables } & \multicolumn{2}{|c|}{ Responder group $(n=23)$} & \multicolumn{2}{|c|}{ Non-responder group $(n=6)$} \\
\hline & Baseline & 24 weeks & Baseline & 24 weeks \\
\hline Body weight (kg) & $66.8 \pm 16.3$ & $67.3 \pm 17.4$ & $62.4 \pm 14.8^{*}$ & $64.3 \pm 15.9$ \\
\hline Waist Circumference (cm) & $97.6 \pm 12.2$ & $98.0 \pm 12.1$ & $84.6 \pm 85^{* *}$ & $85.9 \pm 8.4$ \\
\hline Total insulin dose (units/day) & $71.3 \pm 14.9$ & $32.1 \pm 14.0$ & $59.8 \pm 12.9 * *$ & $54.5 \pm 14.7^{\# \#}$ \\
\hline $\mathrm{HbA1c}(\%)$ & $9.5 \pm 1.2$ & $7.8 \pm 1.1$ & $8.6 \pm 1.3^{*}$ & $8.4 \pm 1.1^{\# \#}$ \\
\hline FBG $(\mathrm{mmol} / \mathrm{L})$ & $12.0 \pm 2.7$ & $7.4 \pm 1.4$ & $9.8 \pm 2.1^{*}$ & $9.2 \pm 1.5^{\# \#}$ \\
\hline P2BG (mmol/L) & $16.7 \pm 4.8$ & $9.8 \pm 2.4$ & $16.3 \pm 4.2$ & $15.8 \pm 4.2^{\# \#}$ \\
\hline HMW adiponectin (ug/ml) & $2.89 \pm 1.54$ & $5.79 \pm 1.58$ & $4.74 \pm 1.46^{* *}$ & $5.11 \pm 1.52^{\# \#}$ \\
\hline
\end{tabular}

Normally distributed data expressed as mean \pm standard deviation and non-normally distributed data expressed as median or as numbers and percentages. Non-normally distributed data were logtransformed for use with parametric statistics. HbA1c: Glycosylated hemoglobin; FBG: fasting blood glucose; P2BG: 2-hour postprandial blood glucose; HMW: high-molecular weight; Data are mean \pm SD. ${ }^{*} \mathrm{p}<0.05$ and ${ }^{* *} \mathrm{P}<0.01$ for responder vs. non-responder at baseline; ${ }^{\#} \mathrm{p}<0.05$ and

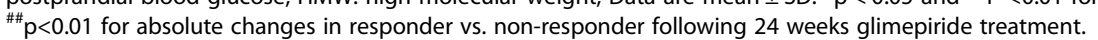

more susceptible to suffer from abdominal obesity compared with European and American subjects [23]. In our study, $75 \%$ subjects with poorly controlled glycemia had abdominal obesity with large dosage of insulin. In obese subjects, circulating adiponectin concentrations inversely correlate with visceral fat area, but not with BMI, and subcutaneous fat area [24]. Reduction of visceral fat increases circulating adiponectin levels in the general population and obese individuals [8]. Several clinical trials have shown that glimepiride could increase serum adiponectin levels in T2D [12,13,25]. The HMW adiponectin is the main active form of the hormone and has relevant role in enhancing insulin sensitivity and protecting against diabetes [15]. For the first time, the present study demonstrated that glimepiride combination with insulin therapy could markedly increase serum HMW adiponectin levels in T2D subjects. We also found a significant negative correlation between changes of $\mathrm{HbA1c}$ and increases in HMW adiponectin levels following 24-week glimepiride treatment, suggesting that the greater reduction in $\mathrm{HbAlc}$ is associated with the greater increases in HMW adiponectin levels. This suggests that the reduced glycemic effect of glimepiride may be mediated through up-regulation and increased secretion of HMW adiponectin by adipocytes [25]. Moreover, several studies supported that insulin seems to suppress expression and secretion of adiponectin both in vitro [26] and in vivo studies [27], suggesting that the great reductions in insulin dose by glimepiride treatment may conversely increase plasma HMW adiponectin concentration. Nagasaka et al. [13] suggest that the increase in adiponectinemia by the glimepiride treatment could be, in part, due to an effect of glycemic control. Therefore, the mechanisms of the increased adiponectinemia by glimepiride may be complex and multifactorial.

Hypoglycemia is the most important barrier associated with anti-diabetic treatment. During the 24-week observation period, adding glimepiride treatment is associated with lower hypoglycemic episodes compared with increasing insulin doses to the insulin-based therapy. Moreover, $67 \%$ of the subjects treated with increasing insulin doses experienced at least one episode of hypoglycaemia, while only $28 \%$ of the subjects with adding glimepiride experienced at least one episode of hypogycaemia. Glimepiride is widely used second-generation sulfonylurea purportedly has lower risk of hypoglycaemia and weight gain relative to other medications in the same class $[28,29]$. The risk of hypoglycemia may be dose-dependent; the relationship between dose and glycemic efficacy of sulfonylurea agents was not linear over the therapeutic dose range. In a doserange finding study with glimepiride, a substantial reduction in HbA1c was observed with $1 \mathrm{mg}$ daily, whereas nearly full efficacy was reached with 4- $\mathrm{mg}$ /day dose with an additional efficacy obtained by escalation through doses of 8-mg/day [30]. Overall, clinical AE and drug-related AEs were reported more frequently with higher doses glimepiride.

Weight gain is another problem associated with insulin treatment. As reported by the UKPDS, subjects with T2D gained about $4 \mathrm{~kg}$ body weight after 10 years of insulin treatment [2]. As expected, in the study, the mean body weight was slightly increased in the group B after 24 weeks insulin administration. The gain in body weight associated with insulin and sulfonylurea agents is an undesirable side effect in subjects with T2D. In the study, we surprisingly found no significant weight gain with glimepiride-added to insulin therapy, which maybe benefit from remarkably decreased insulin doses. Obesity correlates with diabetogenic, atherogenic, pro-thrombolic, and pro-inflammatory metabolic CVDs, which increase the risk of atherosclerotic CVD [23]. Even a slight increase in body weight appears harmful, as it is associated with increased mortality and serious comorbidities, such as hypertension, hyperlipidemia, and CVD. Therefore, the subjects in the group B seem to have greater risk of severe metabolic abnormalities. 


\section{Conclusions}

In summary, we firstly showed in this study that adding glimepiride led to better improvement in glycemic control with a significant smaller daily insulin dose, especially in the abdominal obese T2D subjects with lower HMW adiponectin level before treatment. Most importantly, there was a significant negative correlation between the increase in the HMW adiponectin levels and changes in the HbA1c following glimepiride treatment. And the HMW adiponectin level increased particularly more in responder subjects compared with non-responder subjects, suggesting that increases in the serum HMW adiponectin level may directly contribute to improvement glycemic control. A limitation of the study was the relatively small sample size and would require further research to prove our results.

\section{Competing interests}

The authors declare that they have no competing interests.

\section{Authors' contributions}

DMY and QMZ conceived the study, analyzed data and wrote the manuscript. CJL and JYZ acquired and analyzed data, and wrote the manuscript. All authors read and approved the final manuscript.

\section{Acknowledgments}

This work was supported by the National Nature Science Foundation of China No. 81300663 (to C.J.L.), grants from Tianjin Health Bureau Technology Fund No. 2013 KZ098 (to C.J.L.) and grants from Science and Technology Development Foundation of Tianjin Advanced College No. 2009KY17 (to Q.M.Z.). The authors would like to thank all the subjects and investigators for their participation in this study.

Received: 20 September 2013 Accepted: 14 March 2014

Published: 20 March 2014

\section{References}

1. Heller S: Weight gain during insulin therapy in patients with type 2 diabetes mellitus. Diab Res Clin Pract 2004, 65S:S23-S27.

2. United Kingdom Prospective Diabetes Study: Intensive blood-glucose control with sulphonylureas or insulin compared with conventional treatment and risk of complications in patients with type 2 diabetes (UKPDS 33). Lancet 1998, 352:837-853.

3. Rossetti L, Giaccari A, DeFronzo RA: Glucose toxicity. Diabetes Care 1990, 13(6):610-630.

4. Kahn SE, Haffner SM, Heise MA, Herman WH, Holman RR, Jones NP, Kravitz BG, Lachin JM, O'Neill MC, Zinman B, Viberti G, ADOPT Study Group: Glycemic durability of rosiglitazone, metformin or glyburide monotherapy. N Engl J Med 2006, 355(23):2427-2443.

5. Lincoff AM Wolski K, Nicholls SJ, Nissen SE: Pioglitazone and risk of cardiovascular events in patients with type 2 diabetes mellitus. JAMA 2007, 12; 298:1180-1188.

6. Holden SE, Currie CJ: Dogenous hyperinsulinaemia and exogenous insulin: a common theme between atherosclerosis, increased cancer risk and other morbidities. Atherosclerosis 2012, 222(1):26-28.

7. van Dieren S1, Czernichow S, Chalmers J, Kengne AP, de Galan BE, Poulter N, Woodward M, Beulens JW, Grobbee DE, van der Schouw YT, Zoungas S: Weight changes and their predictors amongst 11140 patients with type 2 diabetes in the ADVANCE trial. Diabetes Obes Metab 2012, 14(5):464-469.

8. Inukai K, Watanabe M, Nakashima Y, Takata N, Isoyama A, Sawa T, Kurihara S, Awata T, Katayama S: Glimepiride enhances intrinsic peroxisome proliferator-activated receptor-gamma activity in 3T3-L1 adipocytes. Biochem Biophys Res Commun 2005, 328(2):484-490.

9. Weyer C, Funahashi T, Tanaka S, Hotta K, Matsuzawa Y, Pratley RE, Tataranni PA: Hypoadiponectinemia in obesity and type 2 diabetes: close association with insulin resistance and hyperinsulinemia. J Clin Endocrinol Metab 2001, 86(5):1930-1935.

10. Waki H, Yamauchi T, Kamon J, Ito Y, Uchida S, Kita S, Hara K, Hada Y, Vasseur F, Froguel P, Kimura S, Nagai R, Kadowaki T: Impaired multimerization of human adiponectin mutants associated with diabetes. Molecular structure and multimer formation of adiponectin. J Biol Chem 2003, 278(41):40352-40363.

11. Kanda Y, Matsuda M, Tawaramoto K, Kawasaki F, Hashiramoto M, Matsuki M, Kaku K: Effects of sulfonylurea drugs on adiponectin production from 3 T3-L1 adipocytes: implication of different mechanism from pioglitazone. Diabetes Res Clin Pract 2008, 81(1):13-18.

12. Tsunekawa T, Hayashi T, Suzuki Y, Matsui-Hirai H, Kano H, Fukatsu A, Nomura N Miyazaki A, Iguchi A: Plasma adiponectin plays an important role in improving insulin resistance with glimepiride in elderly type 2 diabetic subjects. Diabetes Care 2003, 26:285-289.

13. Nagasaka S, Taniguchi A, Aiso Y, Yatagai T, Nakamura T, Nakai Y, Fukushima M, Kuroe A, Ishibashi S: Effect of glimepiride on serum adiponectin level in subjects with type 2 diabetes. Diabetes Care 2003, 26:2215-2216.

14. Fukuen S, Iwaki M, Yasui A, Makishima M, Matsuda M, Shimomura I: Sulfonylurea agents exhibit peroxisome proliferator-activated receptor gamma agonistic activity. J Biol Chem 2005, 280:23653-23659.

15. Liu M, Liu F: Regulation of adiponectin multimerization, signaling and function. J Clin Invest 2006, 116(7):1784-1792.

16. Aso Y, Hara K, Ozeki N, Yatsuka C, Nakano T, Matsumoto S, Suetsugu M, Nakamachi T, Takebayashi K, Haruki K, Inukai T: Low-dose pioglitazone increases serum high molecular weight adiponectin and improves glycemic control in Japanese patients with poorly controlled type 2 diabetes. Diabetes Res Clin Pract 2009, 85(2):147-152.

17. Aso $Y$, Yamamoto $R$, Suetsugu M, Matsumoto S, Wakabayashi S, Matsutomo $R$, Takebayashi K, Inukai T: Comparison of the effects of pioglitazone and voglibose on circulating total and high-molecular-weight adiponectin, and on two fibrinolysis inhibitors, in patients with Type 2 diabetes. Diabet Med 2007, 24(9):962-968.

18. Ose H1, Fukui M, Kitagawa Y, Hirata C, Ichio N, Kadono M, Mogami S, Onishi M, Ichida Y, Nakajima T, Hasegawa G, Yoshikawa T, Nakamura N: Efficacy of glimepiride in patients with poorly controlled insulin-treated type 2 diabetes mellitus. Endocr J 2005, 52:563-569.

19. Riddle MC, Schneider J: The glimepiride combination group beginning insulin treatment of obese patients with evening 70/30 insulin plus glimepiride versus insulin alone. Diabetes Care 1998, 21:1052-1057.

20. Johnson JL, Wolf SL, Kabadi UM: Efficacy of insulin and sulfonylurea combination therapy in type II diabetes. A meta-analysis of the randomized placebo-controlled trials. Arch Intern Med 1996, 12; 156(3):259-264.

21. Mocanu MM, Maddock HL, Baxter GF, Lawrence CL, Standen NB, Yellon DM: Glimepiride, a novel sulfonylurea, does not abolish myocardial protection afforded by either ischemic preconditioning or diazoxide. Circulation 2001, 103(25):3111-3116.

22. Pantalone KM, Kattan MW, Yu C, Wells BJ, Arrigain S, Jain A, Atreja A, Zimmerman RS: The risk of overall mortality in patients with type 2 diabetes receiving glipizide, glyburide, or glimepiride monotherapy: a retrospective analysis. Diabetes Care 2010, 33(6):1224-1229.

23. Kishida K, Funahashi T, Shimomura I: Clinical importance of assessment of type 2 diabetes mellitus with visceral obesity. A Japanese perspective. Curr Diabetes Rev 2012, 8:84-91.

24. Okauchi Y, Kishida K, Funahashi T, Noguchi M, Ogawa T, Ryo M, Okita K, Iwahashi H, Imagawa A, Nakamura T, Matsuzawa Y, Shimomura I: Changes in serum adiponectin concentrations correlate with changes in $\mathrm{BMI}$, waist circumference, and estimated visceral fat area in middle-aged general population. Diabetes Care 2009, 32:e122.

25. Nagasaka $S$, Taniguchi A, Aiso $Y$, Yatagai $T$, Nakamura $T$, Nakai $Y$ : Glimepiride and serum adiponectin level in type 2 diabetic subjects. Diabetes Care 2003, 26:3361.

26. Kadowaki T, Yamauchi T, Kubota N, Hara K, Ueki K, Tobe K: Adiponectin and adiponectin receptors in insulin resistance, diabetes, and the metabolic syndrome. J Clin Invest 2006, 116(7):1784-1792.

27. Yu JG, Javorschi S, Hevener AL, Kruszynska YT, Norman RA, Sinha M, Olefsky JM: The effect of thiazolidinediones on plasma adiponectin levels in normal, obese, and type 2 diabetic subjects. Diabetes 2002, 51:2968-2974 
28. Davis SN: The role of glimepiride in the effective management of type 2 diabetes. J Diabetes Complications 2004, 18:367-376.

29. Korytkowski MT: Sulfonylurea treatment of type 2 diabetes mellitus: focus on glimepiride. Pharmacotherapy 2004, 24:606-620.

30. Miettinen O, Nurminen M: Comparative-analysis of 2 rates. Stat Med 1985 4:213-226.

doi:10.1186/1758-5996-6-41

Cite this article as: Li et al: Adding glimepiride to current insulin therapy increases high-molecular weight adiponectin levels to improve glycemic control in poorly controlled type 2 diabetes. Diabetology \& Metabolic Syndrome 2014 6:41

\section{Submit your next manuscript to BioMed Central and take full advantage of:}

- Convenient online submission

- Thorough peer review

- No space constraints or color figure charges

- Immediate publication on acceptance

- Inclusion in PubMed, CAS, Scopus and Google Scholar

- Research which is freely available for redistribution 